\title{
Endoplasmic reticulum stress and the protein degradation system in ophthalmic diseases
}

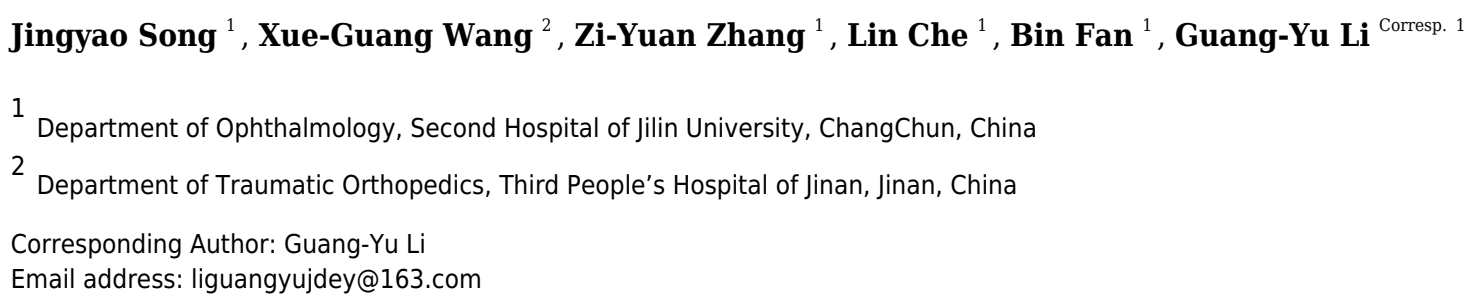

Objective: Endoplasmic reticulum (ER) stress is involved in the pathogenesis of various ophthalmic diseases, and ER stress-mediated degradation systems play an important role in maintaining ER homeostasis during ER stress. The purpose of this review is to explore the potential relationship between them and to find their equilibrium sites. Design: This review illustrates the important role of reasonable regulation of the protein degradation system in ER stress-mediated ophthalmic diseases. There were 128 articles chosen for review in this study, and the keywords used for article research are ER stress, autophagy, UPS, ophthalmic disease, and ocular. Data sources: The data are from Web of Science, PubMed, with no language restrictions from inception until 2019 Jul. Results: The ubiquitin proteasome system (UPS) and autophagy are important degradation systems in ER stress. They can restore ER homeostasis, but if ER stress cannot be relieved in time, cell death may occur. However, they are not independent of each other, and the relationship between them is complementary. Therefore, we propose that ER stability can be achieved by adjusting the balance between them. Conclusion: The degradation system of ER stress, UPS and autophagy are interrelated. Because an imbalance between the UPS and autophagy can cause cell death, regulating that balance may suppress ER stress and protect cells against pathological stress damage. 


\section{Endoplasmic reticulum stress and the protein degradation system in}

\section{2 ophthalmic diseases}

3 Jing-Yao Song ${ }^{1}$, Xue-Guang Wang ${ }^{2}$, Zi-Yuan Zhang ${ }^{1}$, Lin Che ${ }^{1}$,Bin Fan ${ }^{1}$, Guang-Yu Li*

4

5

6

7

8

9

10

11

12

13

14

15

16

17

18

19

20

21

22

23

24

25

26

27

28

29

30

31

32

33

34

35

36

37

38

39

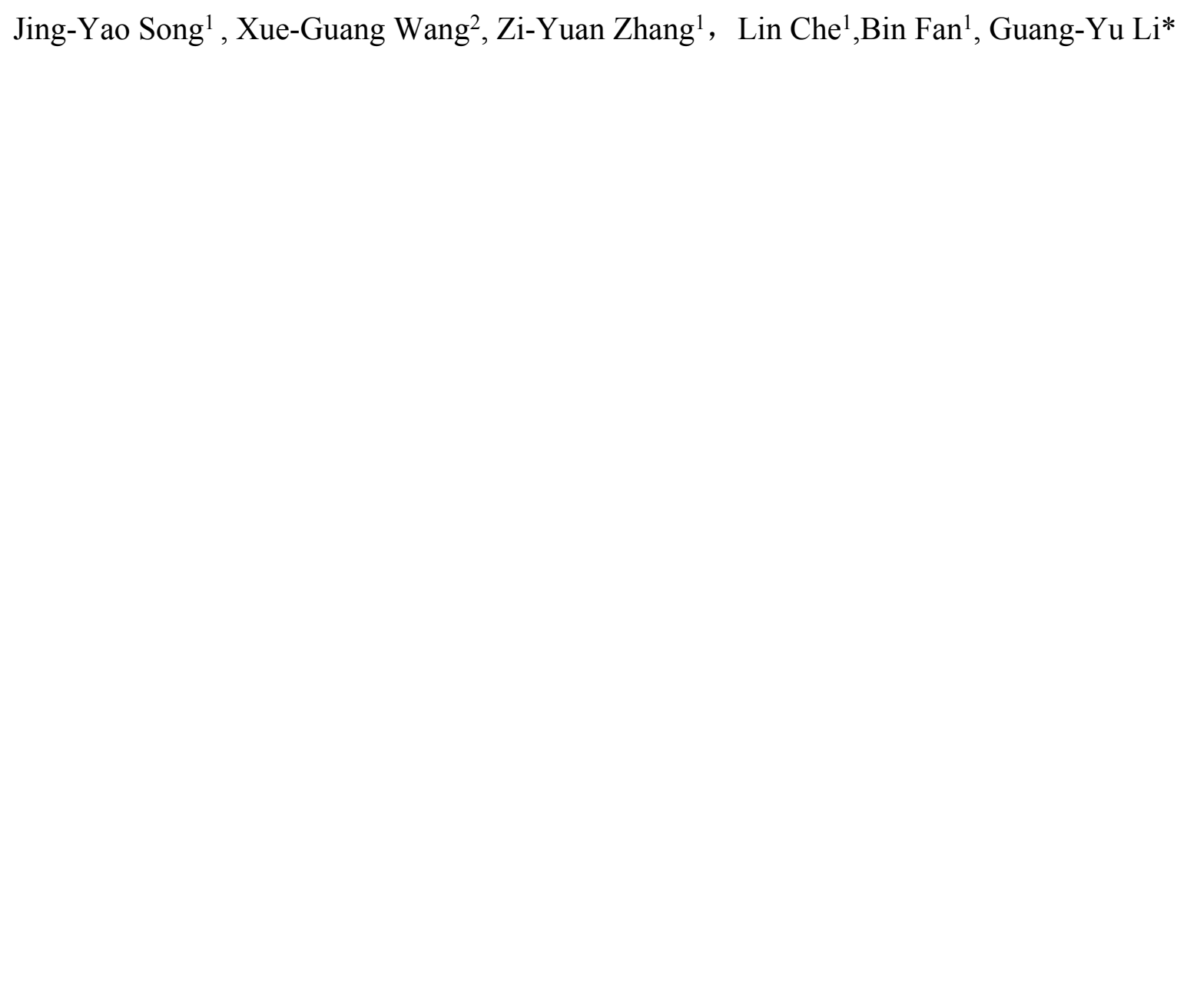

Jing-Yao Song 1 , MS, Department of Ophthalmology, Second Hospital of Jilin University, Changchun 130041, China (1694250634@qq.com)

Xue-Guang Wang ${ }^{2}$, MS, Department of Traumatic Orthopedics, Third People's Hospital of Jinan, Jinan 250113,China (971336377@qq.com)

Zi-Yuan Zhang ${ }^{1}$, MS, Department of Ophthalmology, Second Hospital of Jilin University, Changchun 130041, China (ziyuan.zhang96@outlook.com)

Lin Che ${ }^{1}$, MS, Department of Ophthalmology, Second Hospital of Jilin University, Changchun 130041, China (245431233@qq.com)

Bin Fan ${ }^{1}, \mathrm{PhD}$, Department of Ophthalmology, Second Hospital of Jilin University, Changchun 130041, China (fanbins@qq.com)

*Correspondence: Guang-Yu Li, PhD, Department of Ophthalmology, Second Hospital of Jilin University, Changchun 130041,China(e-mail:liguangyu@aliyun.com) 
40 Abstract

41 Objective: Endoplasmic reticulum (ER) stress is involved in the pathogenesis of various

42

43

44

45 ophthalmic diseases, and ER stress-mediated degradation systems play an important role in maintaining ER homeostasis during ER stress. The purpose of this review is to explore the potential relationship between them and to find their equilibrium sites.

Design: This review illustrates the important role of reasonable regulation of the protein degradation system in ER stress-mediated ophthalmic diseases. There were 128 articles chosen for review in this study, and the keywords used for article research are ER stress, autophagy, UPS, ophthalmic disease, and ocular.

Data sources: The data are from Web of Science, PubMed, with no language restrictions from inception until $2019 \mathrm{Jul}$.

Results: The ubiquitin proteasome system (UPS) and autophagy are important degradation systems in ER stress. They can restore ER homeostasis, but if ER stress cannot be relieved in time, cell death may occur. However, they are not independent of each other, and the relationship between them is complementary. Therefore, we propose that ER stability can be achieved by adjusting the balance between them.

Conclusion: The degradation system of ER stress, UPS and autophagy are interrelated. Because an imbalance between the UPS and autophagy can cause cell death, regulating that balance may suppress ER stress and protect cells against pathological stress damage. 


\section{Introduction}

72 The endoplasmic reticulum (ER) is a highly dynamic and important organelle of eukaryotic cells

73 that has many functions, such as mediating free calcium storage, regulating lipid/sterol synthesis,

74 and participating in the synthesis, processing, and transportation of proteins(Fregno \& Molinari

75 2018). When the internal environment of the ER is destroyed, the accumulation of improperly

76 folded proteins therein eventually leads to ER stress(Li et al. 2017a). In order to inhibit ER stress

77 and coordinate the recovery of ER function, cells have integrated signaling systems, including unfolded protein response (UPR) and the ER-associated degradation (ERAD) pathway(Fujita et al. 2007).

During ER stress, the UPR is activated and performs physiological functions that include enhancement of protein folding ability, stasis of translation of most proteins, and acceleration of protein degradation(Kroeger et al. 2019; Labbadia \& Morimoto 2015). Moreover, ERAD pathways composed by ubiquitin (Ub)-proteasome-dependent and autophagy-lysosomedependent ERAD are also activated to participate in the removal of improperly folded proteins in order to restore the function of the ER(Schroder \& Kaufman 2005a).

Although ER stress, autophagy, and the ubiquitin proteasome system (UPS) have been fully studied in ophthalmic diseases, the relationship among them requires further examination. The purpose of this article is to explore the relationship between them in order to find new opportunities for future research directions and treatment of diseases. We suggest that diseases caused by ER stress can be blocked by regulating the balance between autophagy and the UPS, and especially by removing the pathogenic factor that results in ER stress that cannot be 
92

93

94

95

effectively removed.

\section{Survey methodology}

This review focuses on the relationship between ER stress, autophagy, and the UPS and their interactions in ocular diseases. Academic articles were searched in journal databases such as Web of Science, PubMed, and ER stress, autophagy, UPS, ophthalmic disease, and ocular were the search terms used for article research. The inclusion criteria for the selected articles required that articles be related to ophthalmic diseases and focused on the relationship between ER stress, autophagy, or the UPS.

\section{ER stress and the UPR}

The ER is an important site for protein synthesis, and therefore, if the function of the ER is disrupted by various pathological factors, the excessive accumulation of unfolded or misfolded proteins in the ER may eventually lead to ER stress. Many pathological conditions can lead to ER stress, such as hypoxia, oxidative stress, aging, or metabolic disorders(Lenox et al. 2015; Rozpedek et al. 2016; Rutkowski \& Hegde 2010; Zhu et al. 2018). When ER stress happens, the UPR is activated as a protective mechanism to restore the balance of the ER environment. The UPR involves 3 ER transmembrane proteins: activated transcription factor 6 (ATF6), ER to nuclear signaling 1 (ERN1; also known as inositol-requiring enzyme 1 [IRE1]), and eukaryotic translation initiation factor 2-kinase 3 (EIF2AK3; also known as protein kinase R-like endoplasmic-reticulum kinase [PERK])(Ron \& Walter 2007). Under normal physiological conditions, these proteins bind to the $78 \mathrm{kDa}$ glucose regulatory protein (Grp78; also known as binding immunoglobulin protein $[\mathrm{BiP}])$ in the ER with lumen. During ER stress, BiP is separated 
113 from the sensor so that the UPR signals become activated. IRE1 then becomes phosphorylated,

114 which activates endoribonuclease activity, splices the 26-nucleotide (nt) sequence from X-box

115 binding protein (XBP1) messenger ribonucleic acid (mRNA) and produces functional XBP1(S),

116 which is transferred to the nucleus and activates transcription of the genes encoding the ER

117 chaperone and ERAD(Wakabayashi \& Yoshida 2013). PERK is phosphorylated and activated,

118 which in turn phosphorylates eukaryotic translation initiation factor 2 (eIF2), thereby inhibiting

119 protein translation and reducing protein synthesis(Dan et al. 2017). ATF6 is transported to the

120 Golgi in the form of vesicles and is then cleaved by protease (site 1 and site 2 protease [S1P and

$121 \mathrm{~S} 2 \mathrm{P}]$ ) to produce a transcriptionally active polypeptide(Chen et al. 2002). Activated ATF6

122 translocates to the nucleus and activates the gene transcription of proteins such as ER chaperones

123 that increase ER protein folding(Yamamoto et al. 2007).

124 Interestingly, recent studies have shown that the 3 signaling pathways of the ER transmembrane

125 proteins may interfere with each other. For example, the IRE1 $\alpha$ and PERK pathways are not

126 mutually independent, because knocking out IRE1 $\alpha$ can alter the PERK pathway and also lead to

127 decreased eIF2 $\alpha$ expression(Storniolo et al. 2018). ATF6 is associated with IRE1, and ATF6

128 knockdown can result in unchecked IRE1 reporter activity that increases the splicing of

129 XBP1(Franziska et al. 2018).

130 ER stress and the UPS

131 The UPS is an important protein degradation pathway in eukaryotic cells. Protease and ubiquitin

132 (Ub) in collaboration are responsible for non-lysosomal protein hydrolysis, which may remove

abnormal proteins and prevent the accumulation of nonfunctional and harmful proteins in cells, 
134 therefore maintaining cellular homeostasis(Angele et al. 1999; Coux et al. 1996). The UPS may

135 participate in a variety of biological processes, such as cell cycle, transcription, signaling,

136 trafficking, and protein quality control(Jiang et al. 2018; Rousseau \& Bertolotti 2018). Ub, the

137 smallest protein found in all eukaryotic cells $(8 \mathrm{kDa})$, covalently conjugates many proteins to

label them for downstream effector recognition(Cohen-Kaplan et al. 2017). Ub plays an

important role in cells, including DNA repair, kinase activation, secretion, and protein transport

in endocytic pathways(Kostova et al. 2007).

The conjugation of $\mathrm{Ub}$ protein with a substrate is a multi-step reaction that requires the

participation of many enzymes and consumes energy(Glickman \& Ciechanover 2002; Pickart \&

Eddins 2004). Targeted proteins undergo Ub-proteasome degradation by the 26S proteasome,

which is highly conserved as a 2.5-MDa complex responsible for selective ATP-dependent

degradation of ubiquitinated proteins in eukaryotic cells(Zwickl et al. 1999). It is composed of

2 large subcomplexes consisting of a 28 -subunit 20S protease and a 19-subunit PA700

compound (also called a 19S complex or an S-regulating particle)(Liu \& Jacobson 2013). The

20S proteasome is responsible for substrate degradation, and its 19S subunit assists in

degradation primarily by recognizing the substrate(Glickman 2000). The 19S complex plays an

important role in processing ubiquitinated substrates because it binds, ubiquitinates, and unfolds

ubiquitinated protein, which is then transferred to the proteolysis chamber of the $20 \mathrm{~S}$ proteasome

152 for degradation(Zwickl et al. 1999).

As an important branch of the ERAD pathway, most of the unfolded and misfolded proteins in

the ER are degraded by the UPS pathway(Nakatsukasa \& Brodsky 2008; Zattas \& Hochstrasser

Peer] reviewing PDF | (2019:10:41914:1:1:NEW 16 Jan 2020) 
155 2015). The UPS plays an important role in maintaining ER homeostasis, and impaired Ub-

156 proteasome function can lead to ER stress(Shruthi et al. 2016). Because the UPS can remove

157 proteins from the ER, it is vital to be able to correctly identify improperly folded proteins in the

158 ER. There are at least two monitoring mechanisms for ER protein folding, consisting of one for

159 the luminal domain (soluble or membrane proteins), and the other for the cytoplasmic domain

160 (membrane proteins)(Vashist \& Ng 2004). The UPS removes proteins in the ER through four

161 tightly coupled steps: (1) substrate selection, (2) retro translocation to the cytosol, (3) C-

162 conjugated covalent polyubiquitination, and (4) proteasome degradation(Olzmann et al. 2013).

163 Substrate degradation begins with molecular chaperones, which identify proteins to be degraded

164 by detecting abnormal disulfide bonds or hydrophobic fragments exposed by unassembled

165 protein complexes(Vembar \& Brodsky 2008). In addition, another marker used to identify

166 misfolded proteins is the presence of high mannose (Man5-8GlcNAc2) glycan(Mallinger et al.

167 2012). The substrates are targeted to the retrotranslocation machinery and then translocated to the

168 cytoplasm through the retrotranslocation channel(Vembar \& Brodsky 2008).

169 In addition, ubiquitination is a complex process that requires the participation of three enzymes:

170 ubiquitin activating enzyme E1 (ubiquitin activating enzyme), ubiquitin binding enzyme E2

171 (ubiquitin-conjugating enzyme, E2), and E3 (ubiquitin ligase)(Pickart 2001). At first, E1 forms a

172 high-energy thioester bond with ubiquitin in an ATP-dependent manner, activating the ubiquitin

173 molecule. Then, the activated ubiquitin molecule is transported to E2, acquires the function of

174 recognizing the target protein, and finally binds the target protein under the catalysis of

175 E3(Glickman \& Ciechanover 2002). After repeated enzymatic reactions, the polyubiquitin chain

Peer] reviewing PDF | (2019:10:41914:1:1:NEW 16 Jan 2020) 
176

177

178

179

180

181

182

183

184

185

186

187

binds to the target protein, which is recognized by the $26 \mathrm{~S}$ proteasome, and then

degraded(Hershko \& Ciechanover 1998; Voges et al. 1999).

There are many types of E2 enzymes involved in ubiquitination, but not all E2 types are involved in ERAD. The three types involved in ERAD are ubiquitin-conjugating enzymes J1, J2, and G2 (UBE2J1, UBE2J2, and UBE2G2, respectively)(Christianson \& Ye 2014). Similarly, not all E3 enzymes are involved in ERAD. In yeast, Doa10p and Hrd1p are E3 ligases, and they have participated in the degradation of all substrates that have been studied(Cui et al. 2012). However, E3 ligases found in mammals, such as Hrd1/synoviolin, gp78, TEB4/MARCHVI, RNF5, HRD1, RNF-12, and RNF185, are involved in protein degradation in the ERAD pathway(Darom et al. 2010; El Khouri et al. 2013; You et al. 2016). It was reported that the UPS is regulated by the UPR pathway; for example, the PERK signal can increase the expression of RNF-121 to further enhance the UPS during ER stress(Darom et al. 2010). In addition, XBP1, the downstream factor of IRE1, is required for Nrf2 expression which is the central regulator of cell-protective genes ubiquitous expressed in cells, while Nrf2 interacts with the Cullin3-based E3 ubiquitin ligase adaptor to promote the proteasome(Chen et al. 2018; Ding et al. 2017; Tonelli et al. 2018). This indicates that the Nrf2 factor may be a link by which the IRE1 pathway regulates the UPS.

Moreover, rapamycin is the core of proteasome assembly regulation, while ATF6 is essential for mediating ER stress to activate the mammalian target of the rapamycin (mTOR) pathway(Dylan et al. 2018). Therefore, mTOR may be the intermediate factor for ATF6 to activate UPS.

\section{ER stress and autophagy}

Autophagy is another metabolic pathway that regulates the degradation of long-lived proteins, 
197

198

199

200

201

202

203

204

205

206

207

208

209

210

211

212

213

214

215

216

217

organelles, and other cellular contents(Liu et al. 2015). Autophagy can be divided into

macrophagy, microautophagy, and molecular chaperone-mediated autophagy (CMA)(Bejarano

\& Cuervo 2010; Mijaljica et al. 2011; Parzych \& Klionsky 2014). Macroautophagy is divided

into non-selective and selective autophagy. Non-selective autophagy is usually induced by

nutrient deprivation and often involve the $\mathrm{mTORC} 1$ and protein kinase AMP-activated catalytic

subunit alpha (PRKAA)/adenosine monophosphate-activated protein kinase (AMPK)

pathways(Ganley et al. 2009). Selective autophagy targets specific substrates, including protein

aggregates and damaged organelles such as mitochondria and peroxisomes(Lamark \& Johansen

2012). Macroautophagy is a continuous process involving the formation of autophagosomes, the

fusion of autophagosomes with lysosomes, and the dynamic process of lysosomal

degradation(Baehrecke 2005). It is an important metabolic pathway in eukaryotic cells that is

often used to resist stress and maintain intracellular homeostasis(Boya et al. 2016; Lin \& Kuang

2014; Shi et al. 2013). In the process of microautophagy, the lysosomal membrane acts as a

concave protuberance or membrane, allowing a small portion of the cytoplasmic volume to enter

the lysosomal cavity, which degrades the substrate(Li et al. 2012). Molecular CMA does not

require vacuolar formation and is tightly regulated by chaperone heat shock cognate $71 \mathrm{kDa}$

protein (Hsc70) and its receptor, and it is associated with the PERK pathway in ER stress(Li et

al. 2017b).

Hence, improperly folded proteins are not only degraded through the UPS pathway, but

autophagy is also involved in protein degradation, and an increasing number of studies have

shown that ER stress may trigger autophagy(Bachar-Wikstrom et al. 2013; Chandrika et al.

Peer) reviewing PDF | (2019:10:41914:1:1:NEW 16 Jan 2020) 
218 2015). Misfolded proteins and protein aggregates are cleared under stress by autophagy,

219 especially when the other cellular repair and cellular clearance processes, namely molecular

220 CMA and the UPS, fail(Libby \& Gould 2010; Pandey et al. 2007). In general, ER stress induces

221 autophagy through the IRE1 and PERK signaling pathway(Ogata et al. 2007; Wafa et al. 2013).

222 The downstream factor JNK is activated through the IER1 signaling pathway and further

223 promotes autophagy during ER stress(Gardner \& Walter 2011). Phosphorylated IRE1 also

224 activates the MAPK8/JNK1/MAPK9/MAPK10 pathway, thereby upregulating autophagy(Yan et

225 al. 2018). In addition, spliced XBP1 is reported to be involved in the activation of autophagy by

226 upregulating the transcription of BECN1(Christen \& Fent 2012). The PERK signaling pathway

227 is activated during ER stress, and its downstream factor eIF2a phosphorylates, while

228 phosphorylated eIF2a activates deoxyribonucleic acid (DNA) damage-inducible transcript 3

229 (DDIT3)/ATF4, thereby promoting tribbles pseudokinase 3 (TRIB3) and nuclear protein 1,

230 transcriptional regulator (NUPRl) to induce autophagy by inhibiting Akt1/mTORC1(Salazar M

231 2009; Tang et al. 2015). Additionally, ATF4, which is the downstream factor of PERK, may

232 function as a transcription factor regulating the expression of various autophagy-related

genes(Wafa et al. 2013) (Fig. 1). Studies have shown that ER stress-related autophagy is mainly

mediated by the IRE1a and PERK pathways, while the ATF6 signaling pathway can indirectly

regulate autophagy by upregulating the expression of XBP1 and CHOP.

\section{UPR and ophthalmic diseases}

237 The UPR is known to be an adaptive cellular response to ER dysfunction that suppresses ER 
239

240

241

242

243

244

245

246

247

248

249

250

251

252

253

254

255

256

257

258

259

important role in maintaining the normal physiological function of cells(Sano \& Reed 2013;

Schroder \& Kaufman 2005b). Insufficient activation of the UPR response to ER stress is the

pathogenic factor of age-related retinal neurodegeneration. Thus, the lack of X-box binding

protein 1 (XBP1), which is an important component of the UPR, may accelerate age-related

retinal neurodegenerative diseases(Mclaughlin et al. 2018). It was also reported that in retinal

pigmented epithelium (RPE)-specific XBP1 KO mice showed a 33\% reduction in retinal cone

cells and reduced the thickness of the outer nuclear layer (ONL), suggesting that XBP1 plays an

important role in maintaining ER homeostasis and normal RPE cell function(Zhong et al. 2012).

Moreover, it was shown that PERK activation is a protective response that increases the survival

of photoreceptors in a $\mathrm{P} 23 \mathrm{H}-1$ transgenic rat model, indicating that UPR plays an important role

as the first line of defense against protein-toxic cellular stress(Athanasiou et al. 2017). In

addition, the ATF6 pathway is crucial to human color vision, and ATF6 mutation causes

autosomal-recessive color blindness(Ansar et al. 2015).

UPR plays an important role in maintaining the normal physiological functions of cells under ER stress; however, if UPR still cannot suppress ER stress, it may result in cell death. Thus, if ER stress cannot be effectively controlled, phosphorylated IRE1 $\alpha$ forms a complex with TRAF2 and ASK1 and activates the downstream factor JNK, which may activate caspase-12 to promote apoptosis(Ron \& Hubbard 2008). PERK activation further increases the expression of ATF4 and CHOP, promotes transcription of genes involved in oxidative stress and apoptosis, and further

leads to cell death(Lu \& D. 2004; Rzymski et al. 2009). The activated ATF6 translocates into the nucleus, where it binds the ER stress response elements to activate target genes, including XBP-1 
260

261

262

263

264

265

266

267

268

269

270

271

272

273

274

275

276

277

and CHOP, which directly or indirectly result in cell death(Adachi et al. 2008; Guo et al. 2014;

Hirsch et al. 2014).

An increasing number of studies have shown that ER stress is a factor in the pathology of many ophthalmic diseases, such as chronic glaucoma, glucocorticosteroid-induced glaucoma, cataract, DR, optic-nerve (ON) degeneration, and AMD(Doh et al. 2010; Elmasry et al. 2018; Ojino et al. 2015; Palsamy \& Shinohara 2017; Salminen et al. 2010; Zhou et al. 2016; Zode et al. 2014). For this reason, suppressing ER stress and quickly restoring the ER homeostasis play vital roles in the treatment of those diseases. For example, a recent study indicated that ER stress is detrimental for retinas in the early stages of DR, and suppressing ER stress may protect retinas against visual deficits caused by hyperglycemia(Raji et al. 2018). Moreover, in a mice model of

TON induced by optic nerve crush (ONC), ER stress results in RGC death, however suppressing ER stress through GRP78 overexpression is an effective way to protect RGC from death(Ha et al. 2018). This suggests that reducing the pathological factors that result in ER stress and maintaining cell homeostasis are key factors in sustaining cell physiological functions.

Therefore, UPS and autophagy, as important mechanisms of ERAD, play vital roles in restoring ER homeostasis during ER stress.

\section{UPS and ophthalmic diseases}

The UPS is essential for eye health and is involved in organ development and maintenance of lens function(Liu 2015; Wride \& A. 2011). In addition, it was reported that the proteasome can accelerate protein renewal and efficiently accelerate the degradation of rhodopsin $\mathrm{T} 17 \mathrm{M}$ mutant(Jiang et al. 2014). Inadequate UPS function is involved in the development of ophthalmic 
281 diseases. In glaucoma, decreased ubiquitination in the optic nerve may increase the level of

282 proapoptotic proteins that are normally degraded by proteasomes, leading to axonal degeneration

283 after increased intraocular pressure (IOP)(Dibas et al. 2008). However, overactivation of the

284 UPS also impairs eye health. For example, tumor necrosis factor (TNF) destroys the interstitial

285 connections in human corneal fibroblasts in a manner dependent on the UPS degradation of

286 connexin $43(\mathrm{Cx} 43)$ (Kimura \& Nishida 2010). A study reported that UPS participated in the

287 degradation of rhodopsin and impairs visual function during retinal inflammation(Ozawa et al.

288 2008). Therefore, it was reported that UPS is involved in LPS-induced rat endotoxic uveitis

289 (EIU); intravitreal resolvin D1 (RvD1) can inhibit uveitis by reducing the local level of Ub-

290 proteasome(Rossi et al. 2015).

291 Given that the UPS is an important part of the ERAD pathway, and that it is involved in

removing intracellular proteins, the UPS plays an important role in the inhibition of ER stress-

mediated ophthalmic diseases. The importance of the UPS has been demonstrated in a large

number of diseases associated with protein misfolding(Guerriero \& Brodsky 2012). It was shown

that ER stress is the pathogenic factor involved in granular corneal dystrophy type 2 (GCD2),

while the UPS activated by melatonin can accelerate the degradation of TGF- $\beta$-inducible protein

297 (TGFBI) to suppress ER stress, and thus prevent the death of GCD2 cells(Choi et al. 2017). In

addition, a growing number of studies have shown that if the UPS cannot restore the homeostasis

of ER during ER stress, many ophthalmic diseases may occur. For example, in pseudo- 
302

303

304

305

306

307

308

309

310

311

312

313

314

315

316

317

318

319

320

321

322

patients with high IOP showed the existence of ER stress, and if the UPS cannot inhibit

overactivated ER stress, this could eventually lead to retinal damage(Yang et al. 2015).

\section{Autophagy and ophthalmic diseases}

Under normal physiological conditions, autophagy, which plays an important role in maintaining normal cell function, is maintained at a relatively low level. Neuronal cells, for example, control

cytoskeleton and organelle turnover through the autophagy process, allowing neurons to survive and regenerate after distal axon dissection or nerve suture(David et al. 2015; Tatiana et al. 2018).

Some studies have shown that autophagy deficiency is a pathological factor leading to many

ophthalmic diseases such as corneal opacification, elevated IOP, retinal dystrophy,

mucopolysaccharide storage disease type VI, and AMD(Claudepierre et al. 2010; Golestaneh et

al. 2017; Karnati et al. 2016; Lörincz et al. 2016). Moreover, it was reported that exposure of exvivo mice retinal explants to high glucose resulted in the death of retinal neuronal cells, while treatment the explants with octreotide may protect neuronal cells against high glucose damage by enhancing autophagy(Amato et al. 2018). Although proper autophagy is beneficial to cell survival under stress, overactivated autophagy may lead to cell death, which is called autophagic cell death (ACD)(Liu \& Levine 2015; Vegliante \& Ciriolo 2018). A study demonstrated that the over-activated autophagy lead to the death of photoreceptors and inhibition of autophagy with 3MA may protect photoreceptors against photodamage(Zhang et al. 2014). Thus, as a doubleedged sword, autophagy may either promote cell survival or lead to cell death, depending on the duration and intensity of pathology.

In general, autophagy, as another component mechanism of the ERAD pathway, is a survival 
323

324

325

326

327

328

329

330

331

332

333

334

335

336

337

338

339

340

341

342

mechanism to protect cells against stress, and a large number of studies have shown that autophagy can suppress ER stress and attenuate the pathological damage caused by stress. In glaucoma, enhanced ER stress-mediated autophagy may accelerate myosin clearance in trabecular meshwork cells, thus protecting them against damage. Sulforaphane (SFN) reduces the incidence of posterior cataracts by increasing ER stress-mediated autophagy(Liu et al. 2017). It was also reported that neurons in the lesioned cortex undergo apoptosis after traumatic brain injury, however, treatment with sevoflurane may enhance ER stress-mediated autophagy and inhibit neuronal apoptosis(He et al. 2018). However, ER stress-mediated autophagy also acts as a double-edged sword. For example, It has been shown that in diabetic retinopathy ER stressmediated autophagy caused by a low concentration of oxidized glycosylated low-density lipoprotein (HOG-LDL) may attenuate the loss of peripheral blood cells, while prolonged ER stress-mediated autophagy caused by a higher concentration of HOG-LDL may promote the death of peripheral blood cells(Fu et al. 2016). Hence, excessive ER stress-induced autophagy may also lead to cell death. It was shown that the protective effect of mini- $\alpha \mathrm{A}$ on NaIO3-induced retinal degeneration was achieved by inhibiting ER stress and autophagy(Zhang et al. 2015a). A recent study showed that in a mouse model of retinal degeneration induced by a $\mathrm{P} 23 \mathrm{H}$ rhodopsin gene mutation, the accumulation of misfolded proteins in retinal photoreceptor cells activated ER stress and excessive autophagy, while inhibition of autophagy via deleting the autophagyactivating gene Atg5 decreased photoreceptor death and improved retinal function(Yao et al. 2018). Therefore, whether ER stress-induced autophagy is protective or damaging depends on disease conditions.

Peer) reviewing PDF | (2019:10:41914:1:1:NEW 16 Jan 2020) 
344 The important role of balance between autophagy and UPS during ER stress

345 Both the UPS and autophagy play important roles in maintaining the balance of cellular proteins,

346 and each has its own advantages. The UPS is responsible for the degradation of both short-lived

347 proteins and misfolded proteins, while autophagy can degrade misfolded proteins and damaged

348 organelles(Li et al. 2016). It was found that there is a certain relationship between the UPS and

349 autophagy. It is known that sequestosome 1 (SQSTM1) is a multitasking bridging protein that

350 regulates multiple signaling pathways, and the UPS and autophagy are correlated with each other

through P62 protein(Jorge et al. 2009; Milan et al. 2015). In addition to p62, other adaptors, such

as neighbors of type 1 breast cancer (NBR1), can also recognize ubiquitinated substrates and

localize them to autophagosomes(Cohen-Kaplan et al. 2016). In general, Ub ligase E3 is mainly

degraded and regulated by proteasomes or by the recycling of its own ubiquitination. However, a

recent study demonstrated that etoposide-induced protein 2.4 homolog (EI24) recognizes the

RING domain existing in most E3 ligases and degrades them via the autophagic pathway(de Bie

\& Ciechanover 2011; Nam et al. 2017). In addition, autophagic inhibition impairs the UPS

function and leads to ER stress(Zhang et al. 2015b). It was shown that the functions of autophagy

and the UPS are complementary in some conditions, and passive regulation of the functions

between them is necessary to maintain cell protein homeostasis (Jung et al. 2019)(Fig. 2).

A growing number of studies have shown that the UPS and autophagy may restore cellular 
365

366

367

368

369

370

371

372

373

374

375

376

377

378

379

380

381

382

383

384

385

addition, it was reported that inhibition of autophagy, especially in the case of adequate nutrition, can enhance the activity of proteasomes, which are activated as a compensatory form of protein degradation(Wang et al. 2013). Moreover, Zacks et al. reported that in a mouse model of retinal degeneration caused by a gene mutation in $\mathrm{P} 23 \mathrm{H}$ rhodopsin, ER stress-related autophagy led to photoreceptor death, while the treatment of $\mathrm{P} 23 \mathrm{H}$ mice with selective phosphodiesterase- 4 inhibitor (rolipram) to increase proteasome activity could effectively inhibit ER stress-related autophagy and reduce the rate of retinal degeneration(Qiu et al. 2019). Therefore, the balance between UPS and autophagy is very important, and these two systems have irreplaceable effects on cellular health.

\section{Conclusion}

Autophagy and the UPS are normal phenomena that manage the health of the living eukaryotic cell. However, deficiency and excessive activation of both autophagy and proteasomes are not conducive to cellular health. Many diseases are related to ER stress, and the UPS and autophagy play an important role in suppressing ER stress and maintaining ER homeostasis. We should take this information into consideration while also removing pathogenic factors, especially pathogenic factors such as genetic diseases that cannot be removed by current medical treatments. Then, we may be able to inhibit diseases by simultaneously regulating autophagy and the UPS in order to achieve intracellular homeostasis.

\section{Acknowledgments}

We thank LetPub (www.letpub.com) for its linguistic assistance during the preparation of this manuscript. 


\section{$386 \quad$ Figure legends}

387 Figure 1: ER stress and its degradation pathways.

388

389

390

391

392

393

394

395

396

397

398

399

400

401

402

403

404

405

406

407

408

409

410

411

412

413

414

415

When ER stress occurs, in order to restore the function of the ER, the UPR is activated, and the

UPS and autophagy are activated to suppress ER stress. However, if they still cannot restore the function of the ER, cell death may result.

\section{Figure 2: ER homeostasis can be achieved by balancing the UPS and autophagy pathways}

\section{during ER stress.}

During ER stress, the UPS and autophagy will be activated to remove harmful substrates such as

misfolded proteins or protein aggregates to maintain the normal function of the ER. The balance

between the UPS and autophagy is extremely important for restoring cell homeostasis.

\section{References}

Adachi Y, Yamamoto K, Okada T, Yoshida H, and Mori K. 2008. ATF6 Is a Transcription Factor Specializing in the Regulation of Quality Control Proteins in the Endoplasmic Reticulum. Cell Structure \& Function 33:75-89.

Amato R, Catalani E, Dal Monte M, Cammalleri M, Di Renzo I, Perrotta C, Cervia D, and Casini G. 2018. Autophagymediated neuroprotection induced by octreotide in an ex vivo model of early diabetic retinopathy. Pharmacol Res 128:167-178.

Angele MK, Smail N, Ayala A, Cioffi WG, Bland KI, and Chaudry IH. 1999. L-arginine: a unique amino acid for restoring the depressed macrophage functions after trauma-hemorrhage. J Trauma 46:34-41.

Ansar M, Santos-Cortez RL, Saqib MA, Zulfiqar F, Lee K, Ashraf NM, Ullah E, Wang X, Sajid S, Khan FS , Amin-ud-Din M; University of Washington Center for Mendelian Genomics, Smith JD, Shendure J, Bamshad MJ, Nickerson DA, Hameed A, Riazuddin S, Ahmed ZM, Ahmad W, Leal SM. 2015. Mutation of ATF6 causes autosomal recessive achromatopsia. Hum Genet 134:941-950.

Athanasiou D, Aguila M, Bellingham J, Kanuga N, Adamson P, and Cheetham ME. 2017. The role of the ER stressresponse protein PERK in rhodopsin retinitis pigmentosa. Hum Mol Genet 26:4896-4905.

Bachar-Wikstrom E, Wikstrom JD, Kaiser N, Cerasi E, and Leibowitz G. 2013. Improvement of ER stress-induced diabetes by stimulating autophagy. Autophagy 9:626-628.

Baehrecke EH. 2005. Autophagy: dual roles in life and death? Nat Rev Mol Cell Biol 6:505-510.

Bejarano E, and Cuervo AM. 2010. Chaperone-Mediated Autophagy. Proceedings of the American Thoracic Society 7:29-39. 
416

417

418

419

420

421

422

423

424

425

426

427

428

429

430

431

432

433

434

435

436

437

438

439

440

441

442

443

444

445

446

447

448

449

450

451

452

453

454

455

456

Boya P, Esteban-Martinez L, Serrano-Puebla A, Gomez-Sintes R, and Villarejo-Zori B. 2016. Autophagy in the eye: Development, degeneration, and aging. Prog Retin Eye Res 55:206-245.

Chandrika BB, Yang C, Ou Y, Feng X, Muhoza D, Holmes AF, Theus S, Deshmukh S, Haun RS, and Kaushal GP. 2015. Endoplasmic Reticulum Stress-Induced Autophagy Provides Cytoprotection from Chemical Hypoxia and Oxidant Injury and Ameliorates Renal Ischemia-Reperfusion Injury. Plos One 10:e0140025.

Chen C, Zhong Y, Wang JJ, Yu Q, Plafker K, Plafker S, and Zhang SX. 2018. Regulation of Nrf2 by X Box-Binding Protein 1 in Retinal Pigment Epithelium. Front Genet 9:658.

Chen X, Shen J, and Prywes R. 2002. The Luminal Domain of ATF6 Senses Endoplasmic Reticulum (ER) Stress and Causes Translocation of ATF6 from the ER to the Golgi. Journal of Biological Chemistry 277:13045-13052.

Choi SI, Lee E, Akuzum B, Jeong JB, Maeng YS, Kim TI, and Kim EK. 2017. Melatonin reduces endoplasmic reticulum stress and corneal dystrophy-associated TGFBlp through activation of endoplasmic reticulum-associated protein degradation. J Pineal Res 63.

Christen V, and Fent K. 2012. Silica nanoparticles and silver-doped silica nanoparticles induce endoplasmatic reticulum stress response and alter cytochrome P4501A activity. Chemosphere 87:423-434.

Christianson JC, and Ye Y. 2014. Cleaning up in the endoplasmic reticulum: ubiquitin in charge. Nat Struct Mol Biol 21:325-335.

Claudepierre T, Paques M, Simonutti M, Buard I, Sahel J, Maue RA, Picaud S, and Pfrieger FW. 2010. Lack of NiemannPick type $C 1$ induces age-related degeneration in the mouse retina. Mol Cell Neurosci 43:164-176.

Cohen-Kaplan V, Ciechanover A, and Livneh I. 2017. Stress-induced polyubiquitination of proteasomal ubiquitin receptors targets the proteolytic complex for autophagic degradation. Autophagy 13:759-760.

Cohen-Kaplan V, Livneh I, Avni N, Cohen-Rosenzweig C, and Ciechanover A. 2016. The Ubiquitin-Proteasome System and Autophagy: Coordinated and Independent Activities. International Journal of Biochemistry \& Cell Biology:S1357272516301935.

Coux O, Tanaka K, and Goldberg AL. 1996. Structure and functions of the $20 \mathrm{~S}$ and $26 \mathrm{~S}$ proteasomes. Annu Rev Biochem 65:801-847.

Cui F, Liu L, Zhao Q, Zhang Z, Li Q, Lin B, Wu Y, Tang S, and Xie Q. 2012. Arabidopsis Ubiquitin Conjugase UBC32 Is an ERAD Component That Functions in Brassinosteroid-Mediated Salt Stress Tolerance. Plant Cell 24:233-244.

Dan L, Laura K, Ove E, and Sulev Kk. 2017. Recent Insights into the Role of Unfolded Protein Response in ER Stress in Health and Disease. Frontiers in Cell \& Developmental Biology 5:48-.

Darom A, Bening-Abu-Shach U, and Broday L. 2010. RNF-121 Is an Endoplasmic Reticulum-Membrane E3 Ubiquitin Ligase Involved in the Regulation of -Integrin. Molecular Biology of the Cell 21:1788-1798.

David K, D. CS, and M. ID. 2015. Decreased Energy Capacity and Increased Autophagic Activity in Optic Nerve Axons With Defective Anterograde Transport. Invest Ophthalmol Vis Sci 56:8215-.

de Bie P, and Ciechanover A. 2011. Ubiquitination of E3 ligases: self-regulation of the ubiquitin system via proteolytic and non-proteolytic mechanisms. Cell Death Differ 18:1393-1402.

Dibas A, Yang MH, He S, Bobich J, and Yorio T. 2008. Changes in ocular aquaporin-4 (AQP4) expression following retinal injury. Mol Vis 14:1770-1783.

Ding H, Wang X, Wang H, Zhu L, Wang Q, Jia Y, Wei W, Zhou C, Wu H, and Ding K. 2017. Nrf2-ARE signaling provides neuroprotection in traumatic brain injury via modulation of the ubiquitin proteasome system. Neurochem Int 111:32-44.

Doh SH, Kim JH, Lee KM, Park HY, and Park CK. 2010. Retinal ganglion cell death induced by endoplasmic reticulum

Peer] reviewing PDF | (2019:10:41914:1:1:NEW 16 Jan 2020) 
457

458

459

460

461

462

463

464

465

466

467

468

469

470

471

472

473

474

475

476

477

478

479

480

481

482

483

484

485

486

487

488

489

490

491

492

493

494

495

496

497

stress in a chronic glaucoma model. Brain Res 1308:158-166.

Dylan, Allen, Jin, and Seo. 2018. ER Stress Activates the TOR Pathway through Atf6. J Mol Signal 13:1.

El Khouri E, Le Pavec G, Toledano MB, and Delaunay-Moisan A. 2013. RNF185 Is a Novel E3 Ligase of Endoplasmic Reticulum-associated Degradation (ERAD) That Targets Cystic Fibrosis Transmembrane Conductance Regulator (CFTR). Journal of Biological Chemistry 288:31177-31191.

Elmasry K, Ibrahim AS, Saleh H, Elsherbiny N, Elshafey S, Hussein KA, and Al-Shabrawey M. 2018. Role of endoplasmic reticulum stress in 12/15-lipoxygenase-induced retinal microvascular dysfunction in a mouse model of diabetic retinopathy. Diabetologia 61:1220-1232.

Franziska, Walter, Aisling, O'Brien, Caoimhín, Concannon, Heiko, Düssmann, and Jochen. 2018. ER stress signalling has an activating transcription factor $6 \alpha$ (ATF6)-dependent 'off-switch'. The Journal of biological chemistry. 293(47):18270-18284

Fregno I, and Molinari M. 2018. Endoplasmic reticulum turnover: ER-phagy and other flavors in selective and nonselective ER clearance. F1000Res 7:454.

Fu D, Yu JY, Yang S, Wu M, Hammad SM, Connell AR, Du M, Chen J, and Lyons TJ. 2016. Survival or death: a dual role for autophagy in stress-induced pericyte loss in diabetic retinopathy. Diabetologia 59:2251-2261.

Fujita E, Kouroku Y, Isoai A, Kumagai H, Misutani A, Matsuda C, Hayashi YK, and Momoi T. 2007. Two endoplasmic reticulum-associated degradation (ERAD) systems for the novel variant of the mutant dysferlin: ubiquitin/proteasome ERAD(I) and autophagy/lysosome ERAD(II). Hum Mol Genet 16:618-629.

Ganley IG, Du HL, Wang J, Ding X, Chen S, and Jiang X. 2009. ULK1·ATG13·FIP200 Complex Mediates mTOR Signaling and Is Essential for Autophagy *. Journal of Biological Chemistry 284.

Gardner BM, and Walter P. 2011. Unfolded Proteins Are Ire1-Activating Ligands That Directly Induce the Unfolded Protein Response. Journal of Cell Science 333:1891-1894.

Glickman MH. 2000. Getting in and out of the proteasome. Semin Cell Dev Biol 11:149-158.

Glickman MH, and Ciechanover A. 2002. The ubiquitin-proteasome proteolytic pathway: destruction for the sake of construction. Physiol Rev 82:373-428.

Golestaneh N, Chu Y, Xiao YY, Stoleru GL, and Theos AC. 2017. Dysfunctional autophagy in RPE, a contributing factor in age-related macular degeneration. Cell Death \& Disease 8:e2537.

Guerriero CJ, and Brodsky JL. 2012. The Delicate Balance Between Secreted Protein Folding and Endoplasmic Reticulum-Associated Degradation in Human Physiology. Physiol Rev 92:537-576.

Guo FJ, Xiong Z, Lu X, Ye M, Han X, and Jiang R. 2014. ATF6 upregulates XBP1S and inhibits ER stress-mediated apoptosis in osteoarthritis cartilage. Cell Signal 26:332-342.

Ha Y, Liu W, Liu H, Zhu S, Xia F, Gerson JE, Azhar NA, Tilton RG, Motamedi M, Kayed R et al. 2018. AAV2-mediated GRP78 Transfer Alleviates Retinal Neuronal Injury by Downregulating ER Stress and Tau Oligomer Formation. Invest Ophthalmol Vis Sci 59:4670-4682.

Hayat B, Padhy B, Mohanty PP, and Alone DP. 2019. Altered unfolded protein response and proteasome impairment in pseudoexfoliation pathogenesis. Exp Eye Res 181:197-207.

He H, Weifeng L, Yingying Z, Yibin L, Peiqing W, Yasong L, and Huangde F. 2018. Sevoflurane post-conditioning attenuates traumatic brain injury-induced neuronal apoptosis by promoting autophagy via the PI3K/AKT signaling pathway. Drug Design Development \& Therapy Volume 12:629-638.

Hershko A, and Ciechanover A. 1998. The ubiquitin system. Annu Rev Biochem 67:425-479.

Hirsch I, Weiwad M, Prell E, and Ferrari DM. 2014. ERp29 deficiency affects sensitivity to apoptosis via impairment

Peer) reviewing PDF | (2019:10:41914:1:1:NEW 16 Jan 2020) 
498

499

500

501

502

503

504

505

506

507

508

509

510

511

512

513

514

515

516

517

518

519

520

521

522

523

524

525

526

527

528

529

530

531

532

533

534

535

536

537

538

of the ATF6-CHOP pathway of stress response. Apoptosis 19:801-815.

Jiang $H$, Xiong S, and Xia X. 2014. Retinitis pigmentosa?associated rhodopsin mutant T17M induces endoplasmic reticulum (ER) stress and sensitizes cells to ER stress-induced cell death. Molecular Medicine Reports 9:1737---1742.

Jiang TX, Zhao M, and Qiu XB. 2018. Substrate receptors of proteasomes. Biol Rev Camb Philos Soc 93:1765-1777.

Jorge, Moscat, and, Maria, T., and Diaz-Meco. 2009. p62 at the Crossroads of Autophagy, Apoptosis, and Cancer. Cell. 137(6):0-1004.

Jung, Hoon, Lee, Seoyoung, Park, Eunkyoung, Kim, Min, and Jae. 2019. Negative-feedback coordination between proteasomal activity and autophagic flux. Autophagy 15(4):726-728.

Karnati R, Talla V, Peterson K, and Laurie GW. 2016. Lacritin and other autophagy associated proteins in ocular surface health. Exp Eye Res 144:4-13.

Kimura K, and Nishida T. 2010. Role of the ubiquitin-proteasome pathway in downregulation of the gap-junction protein Connexin43 by TNF-\{alpha\} in human corneal fibroblasts. Invest Ophthalmol Vis Sci 51:1943-1947.

Kostova Z, Tsai YC, and Weissman AM. 2007. Ubiquitin ligases, critical mediators of endoplasmic reticulumassociated degradation. Semin Cell Dev Biol 18:770-779.

Kroeger H, Chiang WC, Felden J, Nguyen A, and Lin JH. 2019. ER stress and unfolded protein response in ocular health and disease. FEBS J 286:399-412.

Labbadia J, and Morimoto RI. 2015. Repression of the Heat Shock Response Is a Programmed Event at the Onset of Reproduction. Molecular Cell:S1097276515004992.

Lamark T, and Johansen T. 2012. Aggrephagy: selective disposal of protein aggregates by macroautophagy. Int J Cell Biol 2012:736905.

Lenox AR, Bhootada Y, Gorbatyuk O, Fullard R, and Gorbatyuk M. 2015. Unfolded protein response is activated in aged retinas. Neuroscience Letters 609:30-35.

Li F, Yang Y, Yang L, Wang K, Zhang X, Zong Y, Ding Y, Wang C, Zhang L, and Ji G. 2017a. Resveratrol alleviates FFA and $\mathrm{CCl} 4$ induced apoptosis in HepG2 cells via restoring endoplasmic reticulum stress. Oncotarget 8:4379943809.

Li W, Zhu J, Dou J, She H, Tao K, Xu H, Yang Q, and Mao Z. 2017b. Phosphorylation of LAMP2A by p38 MAPK couples ER stress to chaperone-mediated autophagy. Nat Commun 8:1763.

Li WW, Li J, and Bao JK. 2012. Microautophagy: lesser-known self-eating. Cell Mol Life Sci 69:1125-1136.

Li X, Zhu F, Jiang J, Sun C, Zhong Q, Shen M, Wang X, Tian R, Shi C, Xu M, Peng F, Guo X, Hu J, Ye D, Wang M, Qin R. 2016. Simultaneous inhibition of the ubiquitin-proteasome system and autophagy enhances apoptosis induced by ER stress aggravators in human pancreatic cancer cells. Autophagy 12:1521-1537.

Libby RT, and Gould DB. 2010. Endoplasmic Reticulum Stress as a Primary Pathogenic Mechanism Leading to AgeRelated Macular Degeneration. Advances in Experimental Medicine \& Biology 664:403-409.

Lin WJ, and Kuang HY. 2014. Oxidative stress induces autophagy in response to multiple noxious stimuli in retinal ganglion cells. Autophagy 10:1692-1701.

Liu C, DeRoo EP, Stecyk C, Wolsey M, Szuchnicki M, and Hagos EG. 2015. Impaired autophagy in mouse embryonic fibroblasts null for Kruppel-like Factor 4 promotes DNA damage and increases apoptosis upon serum starvation. Mol Cancer 14:101.

Liu CW, and Jacobson AD. 2013. Functions of the 19S complex in proteasomal degradation. Trends Biochem Sci 38:103-110.

Peer] reviewing PDF | (2019:10:41914:1:1:NEW 16 Jan 2020) 
Liu H, Smith AJ, Ball SS, Bao Y, Bowater RP, Wang N, and Michael Wormstone I. 2017. Sulforaphane promotes ER stress, autophagy, and cell death: implications for cataract surgery. J Mol Med (Berl) 95:553-564.

Liu Kea. 2015. Altered ubiquitin causes perturbed calcium homeostasis, hyperactivation of calpain, dysregulated differentiation, and cataract. Proceedings of the National Academy of Sciences of the United States of America 112:1071-1076.

Liu Y, and Levine B. 2015. Autosis and autophagic cell death: the dark side of autophagy. Cell Death Differ 22:367376.

Lőrincz P, Takáts S, Kárpáti M, and Juhász G. 2016. IFly: The eye of the fruit fly as a model to study autophagy and related trafficking pathways. Exp Eye Res 144:90-98.

Lu, and D. P. 2004. Translation reinitiation at alternative open reading frames regulates gene expression in an integrated stress response. Journal of Cell Biology 167:27-33.

Mallinger A, Wen HM, Dankle GM, and Glenn KA. 2012. Using a ubiquitin ligase as an unfolded protein sensor. Biochem Biophys Res Commun 418:0-48.

Mclaughlin T, Falkowski M, Park JW, Keegan S, and Zhang SX. 2018. Loss of XBP1 accelerates age-related decline in retinal function and neurodegeneration. Molecular Neurodegeneration 13:16.

Mijaljica D, Prescott M, and Devenish RJ. 2011. Microautophagy in mammalian cells: Revisiting a 40-year-old conundrum. Autophagy 7:673-682.

Milan E, Perini T, Resnati M, Orfanelli U, Oliva L, Raimondi A, Cascio P, Bachi A, Marcatti M, Ciceri F et al. 2015. A plastic SQSTM1/p62-dependent autophagic reserve maintains proteostasis and determines proteasome inhibitor susceptibility in multiple myeloma cells. Autophagy 11:1161-1178.

Nakatsukasa K, and Brodsky JL. 2008. The recognition and retrotranslocation of misfolded proteins from the endoplasmic reticulum. Traffic 9:861-870.

Nam T, Han JH, Devkota S, and Lee HW. 2017. Emerging Paradigm of Crosstalk between Autophagy and the UbiquitinProteasome System. Moleculer Cells 40:897-905.

Ogata M, Hino SI, Saito A, Morikawa K, and Imaizumi K. 2007. Autophagy Is Activated for Cell Survival after Endoplasmic Reticulum Stress. Molecular and Cellular Biology 26:9220-9231.

Ojino K, Shimazawa M, Izawa H, Nakano Y, Tsuruma K, and Hara H. 2015. Involvement of endoplasmic reticulum stress in optic nerve degeneration after chronic high intraocular pressure in DBA/2J mice. J Neurosci Res 93:1675-1683.

Olzmann JA, Kopito RR, and Christianson JC. 2013. The Mammalian Endoplasmic Reticulum-Associated Degradation System. Cold Spring Harbor Perspectives in Biology 5:a013185-a013185.

Ozawa Y, Nakao K, Kurihara T, Shimazaki T, Shimmura S, Ishida S, Yoshimura A, Tsubota K, and Okano H. 2008. Roles of STAT3/SOCS3 pathway in regulating the visual function and ubiquitin-proteasome-dependent degradation of rhodopsin during retinal inflammation. J Biol Chem 283:24561-24570.

Palsamy P, and Shinohara T. 2017. Age-related cataracts: Role of unfolded protein response, Ca 2+ mobilization, epigenetic DNA modifications, and loss of Nrf2/Keap1 dependent cytoprotection. Progress in Retinal \& Eye Research:S1350946217300575.

Pandey UB, Nie Z, Batlevi Y, McCray BA, Ritson GP, Nedelsky NB, Schwartz SL, DiProspero NA, Knight MA, Schuldiner O , Padmanabhan R, Hild M, Berry DL, Garza D, Hubbert CC, Yao TP, Baehrecke EH, Taylor JP. 2007. HDAC6 rescues neurodegeneration and provides an essential link between autophagy and the UPS. Nature 447:859-863.

PeerJ reviewing PDF | (2019:10:41914:1:1:NEW 16 Jan 2020) 
580

581

582

583

584

585

586

587

588

589

590

591

592

593

594

595

596

597

598

599

600

601

602

603

604

605

606

607

608

609

610

611

612

613

614

615

616

617

618

619

620

Parzych KR, and Klionsky DJ. 2014. An overview of autophagy: morphology, mechanism, and regulation. Antioxid Redox Signal 20:460-473.

Pickart CM. 2001. Mechanisms underlying ubiquitination. Annu Rev Biochem 70:503-533.

Pickart CM, and Eddins MJ. 2004. Ubiquitin: structures, functions, mechanisms. Biochim Biophys Acta 1695:55-72.

Qiu Y, Yao J, Jia L, Thompson DA, and Zacks DN. 2019. Shifting the balance of autophagy and proteasome activation reduces proteotoxic cell death: a novel therapeutic approach for restoring photoreceptor homeostasis. Cell Death Dis 10:547.

Raji L, G. NP, Shanta A, R. RV, A. CM, B. KU, and Rajashekhar G. 2018. Critical role of endoplasmic reticulum stress in chronic endothelial activation?induced visual deficits in tie2-tumor necrosis factor mice. J Cell Biochem 119:8460-8471.

Ron D, and Hubbard SR. 2008. How IRE1 Reacts to ER Stress. Cell 132:0-26.

Ron D, and Walter P. 2007. Signal integration in the endoplasmic reticulum unfolded protein response. Natrevmolcell Biol 8:519-529.

Rossi S, Di Filippo C, Gesualdo C, Potenza N, Russo A, Trotta MC, Zippo MV, Maisto R, Ferraraccio F, Simonelli F , D'Amico M . 2015. Protection from endotoxic uveitis by intravitreal Resolvin D1: involvement of lymphocytes, miRNAs, ubiquitin-proteasome, and M1/M2 macrophages. Mediators Inflamm 2015:149381.

Rousseau A, and Bertolotti A. 2018. Regulation of proteasome assembly and activity in health and disease. Nat Rev Mol Cell Biol 19:697-712.

Rozpedek W, Pytel D, Mucha B, Leszczynska H, Diehl JA, and Majsterek I. 2016. The Role of the PERK/elF2alpha/ATF4/CHOP Signaling Pathway in Tumor Progression During Endoplasmic Reticulum Stress. Curr Mol Med 16:533-544.

Rutkowski DT, and Hegde RS. 2010. Regulation of basal cellular physiology by the homeostatic unfolded protein response. J Cell Biol 189:783-794.

Rzymski T, Milani M, Singleton DC, and Harris AL. 2009. Role of ATF4 in regulation of autophagy and resistance to drugs and hypoxia. Cell Cycle 8:3838-3847.

Salazar M CA, Salanueva IJ, Hernandez-Tiedra S, Lorente M, Egia A, Vazquez P, Blazquez C, Torres S, Garcia S, Nowak J, Fimia GM, Piacentini M, Cecconi F, Pandolfi PP, González-Feria L, lovanna JL, Guzmán M, Boya P, Velasco G. 2009. Cannabinoid action induces autophagy-mediated cell death through stimulation of ER stress in human glioma cells. Journal of Clinical Investigation 119:1359-1372.

Salminen A, Kauppinen A, Hyttinen JM, Toropainen E, and Kaarniranta K. 2010. Endoplasmic reticulum stress in agerelated macular degeneration: trigger for neovascularization. Mol Med 16:535-542.

Sano R, and Reed JC. 2013. ER stress-induced cell death mechanisms. Biochim Biophys Acta 1833:3460-3470.

Schroder M, and Kaufman RJ. 2005a. ER stress and the unfolded protein response. Mutat Res 569:29-63.

Schroder M, and Kaufman RJ. 2005b. The mammalian unfolded protein response. Annu Rev Biochem 74:739-789.

Shi X, Wu YC, Zhu XY, and Sun XD. 2013. [The latest advance of correlation between autophagy and optic neuritis]. [Zhonghua yan ke za zhi] Chinese journal of ophthalmology 49:956-959.

Shruthi K, Reddy SS, Reddy PY, Shivalingam P, Harishankar N, and Reddy GB. 2016. Amelioration of neuronal cell death in a spontaneous obese rat model by dietary restriction through modulation of ubiquitin proteasome system. Journal of Nutritional Biochemistry 33:73-81.

Storniolo A, Alfano V, Carbotta S, Ferretti E, and Di Renzo L. 2018. IRE1 $\alpha$ deficiency promotes tumor cell death and elF2 $\alpha$ degradation through PERK dipendent autophagy. Cell Death Discovery 4:3.

Peer) reviewing PDF | (2019:10:41914:1:1:NEW 16 Jan 2020) 
621

622

623

624

625

626

627

628

629

630

631

632

633

634

635

636

637

638

639

640

641

642

643

644

645

646

647

648

649

650

651

652

653

654

655

656

657

658

659

660

661

Tang B, Jingjing C, Lin S, Yiping L, Jia Q, Joy SB, Shengzhou W, and C. RD. 2014. Proteasome Inhibitors Activate Autophagy Involving Inhibition of PI3K-Akt-mTOR Pathway as an Anti-Oxidation Defense in Human RPE Cells. Plos One 9:e103364-.

Tang B, Li Q, Zhao XH, Wang HG, Li N, Fang Y, Wang K, Jia YP, Zhu P, Gu J, Li JX, Jiao YJ, Tong WD, Wang M, Zou QM, Zhu FC, Mao XH. 2015. Shiga toxins induce autophagic cell death in intestinal epithelial cells via the endoplasmic reticulum stress pathway. Autophagy 11:344-354.

Tatiana LR, David R-G, Sara M-M-A, Mireia H-G, Assumpció B, Joaquim F, and Caty C. 2018. ATG5 overexpression is neuroprotective and attenuates cytoskeletal and vesicle-trafficking alterations in axotomized motoneurons. Cell Death \& Disease 9:626-.

Tonelli C, Chio IIC, and Tuveson DA. 2018. Transcriptional Regulation by Nrf2. Antioxid Redox Signal 29:1727-1745.

Vashist S, and Ng DT. 2004. Misfolded proteins are sorted by a sequential checkpoint mechanism of ER quality control. J Cell Biol 165:41-52.

Vegliante R, and Ciriolo MR. 2018. Autophagy and Autophagic Cell Death: Uncovering New Mechanisms Whereby Dehydroepiandrosterone Promotes Beneficial Effects on Human Health. Vitam Horm 108:273-307.

Vembar SS, and Brodsky JL. 2008. One step at a time: endoplasmic reticulum-associated degradation. Nat Rev Mol Cell Biol 9:944-957.

Voges D, Zwickl P, and Baumeister W. 1999. The 26S proteasome: a molecular machine designed for controlled proteolysis. Annu Rev Biochem 68:1015-1068.

Wafa Bc, Anne-Catherine M, Valérie C, Julien A, Céline J, Yuki M, Laurent P, Georges S, Pierre F, and Alain B. 2013. The elF2 $\alpha /$ ATF4 pathway is essential for stress-induced autophagy gene expression. Nucleic Acids Research:16.

Wakabayashi S, and Yoshida H. 2013. The essential biology of the endoplasmic reticulum stress response for structural and computational biologists. Comput Struct Biotechnol J 6:e201303010.

Wang XJ, Yu J, Wong SH, Cheng AS, Chan FK, Ng SS, Cho CH, Sung JJ, and Wu WK. 2013. A novel crosstalk between two major protein degradation systems. Autophagy 9:1500-1508.

Wride, and A. M. 2011. Lens fibre cell differentiation and organelle loss: many paths lead to clarity. Philosophical Transactions Biological Sciences 366:1219-1233.

Yamamoto K, Sato T, Matsui T, Sato M, Okada T, Yoshida H, Harada A, and Mori K. 2007. Transcriptional Induction of Mammalian ER Quality Control Proteins Is Mediated by Single or Combined Action of ATF6 $\alpha$ and XBP1. Developmental Cell 13:0-376.

Yan B, Yunting P, Xiang Y, D. GB, Renu S, H. HS, and C. BD. 2018. IRE1B degrades RNAs encoding proteins that interfere with the induction of autophagy by $E R$ stress in $\backslash \backslash r \quad<i>$ Arabidopsis thaliana $</ i>$. Autophagy:15548627.15542018.11462426-.

Yang X, Hondur G, Li M, Cai J, and Tezel G. 2015. Proteomics Analysis of Molecular Risk Factors in the Ocular Hypertensive Human Retina. Invest Ophthalmol Vis Sci 56:5816-5830.

Yao J, Qiu Y, Frontera E, Jia L, Khan NW, Klionsky DJ, Ferguson TA, Thompson DA, and Zacks DN. 2018. Inhibiting autophagy reduces retinal degeneration caused by protein misfolding. Autophagy 14:1226-1238.

You, Hui, Shen, Yachen, Yan, Caifeng, Huang, Yujie, Xu, and Weifeng. 2016. HRD1-Mediated IGF-1R Ubiquitination Contributes to Renal Protection of Resveratrol in $\mathrm{db} / \mathrm{db}$ Mice.

Zattas D, and Hochstrasser M. 2015. Ubiquitin-dependent protein degradation at the yeast endoplasmic reticulum and nuclear envelope. Critical Reviews in Biochemistry \& Molecular Biology 50:1-17.

Peer] reviewing PDF | (2019:10:41914:1:1:NEW 16 Jan 2020) 
662

663

664

665

666

667

668

669

670

671

672

673

674

675

676

677

678

679

680

Zhang J, Zhao X, Cai Y, Li Y, Yu X, and Lu L. 2015a. Protection of Retina by Mini-aA in NalO3-Induced Retinal Pigment Epithelium Degeneration Mice. International Journal of Molecular Sciences 16:1644.

Zhang TZ, Fan B, Chen X, Wang W-J, Jiao Y-Y, Su G-F, and Li G-Y. 2014. Suppressing autophagy protects photoreceptor cells from light-induced injury. Biochem Biophys Res Commun 450:966-972.

Zhang Y, Ye M, Chen L, Li M, Tang Z, and Wang C. 2015b. Role of the ubiquitin-proteasome system and autophagy in regulation of insulin sensitivity in serum-starved 3T3-L1 adipocytes. Endocrine Journal 62:673.

Zhong Y, Jingming L, J. WJ, Chen C, A. TJ-T, Anisse S, Qiang Y, Yun-zheng L, A. MMN, and E. AR. 2012. X-Box Binding Protein 1 Is Essential for the Anti-Oxidant Defense and Cell Survival in the Retinal Pigment Epithelium. Plos One 7:e38616-.

Zhou Y, Bennett TM, and Shiels A. 2016. Lens ER-stress response during cataract development in Mip-mutant mice. Biochim Biophys Acta 1862:1433-1442.

Zhu X, Wang K, Zhou F, and Zhu L. 2018. Paeoniflorin attenuates atRAL-induced oxidative stress, mitochondrial dysfunction and endoplasmic reticulum stress in retinal pigment epithelial cells via triggering

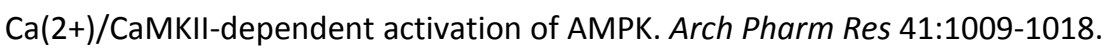

Zode GS, Sharma AB, Lin X, Searby CC, Bugge K, Kim GH, Clark AF, and Sheffield VC. 2014. Ocular-specific ER stress reduction rescues glaucoma in murine glucocorticoid-induced glaucoma. J Clin Invest 124:1956-1965.

Zwickl P, Voges D, and Baumeister W. 1999. The proteasome: a macromolecular assembly designed for controlled proteolysis. Philos Trans R Soc Lond B Biol Sci 354:1501-1511.

Peer) reviewing PDF | (2019:10:41914:1:1:NEW 16 Jan 2020) 
Figure 1

Figure 1: ER stress and its degradation pathways.

When ER stress occurs, in order to restore the function of the ER, the UPR is activated, and the UPS and autophagy are activated to suppress ER stress. However, if they still cannot restore the function of the ER, cell death may result.

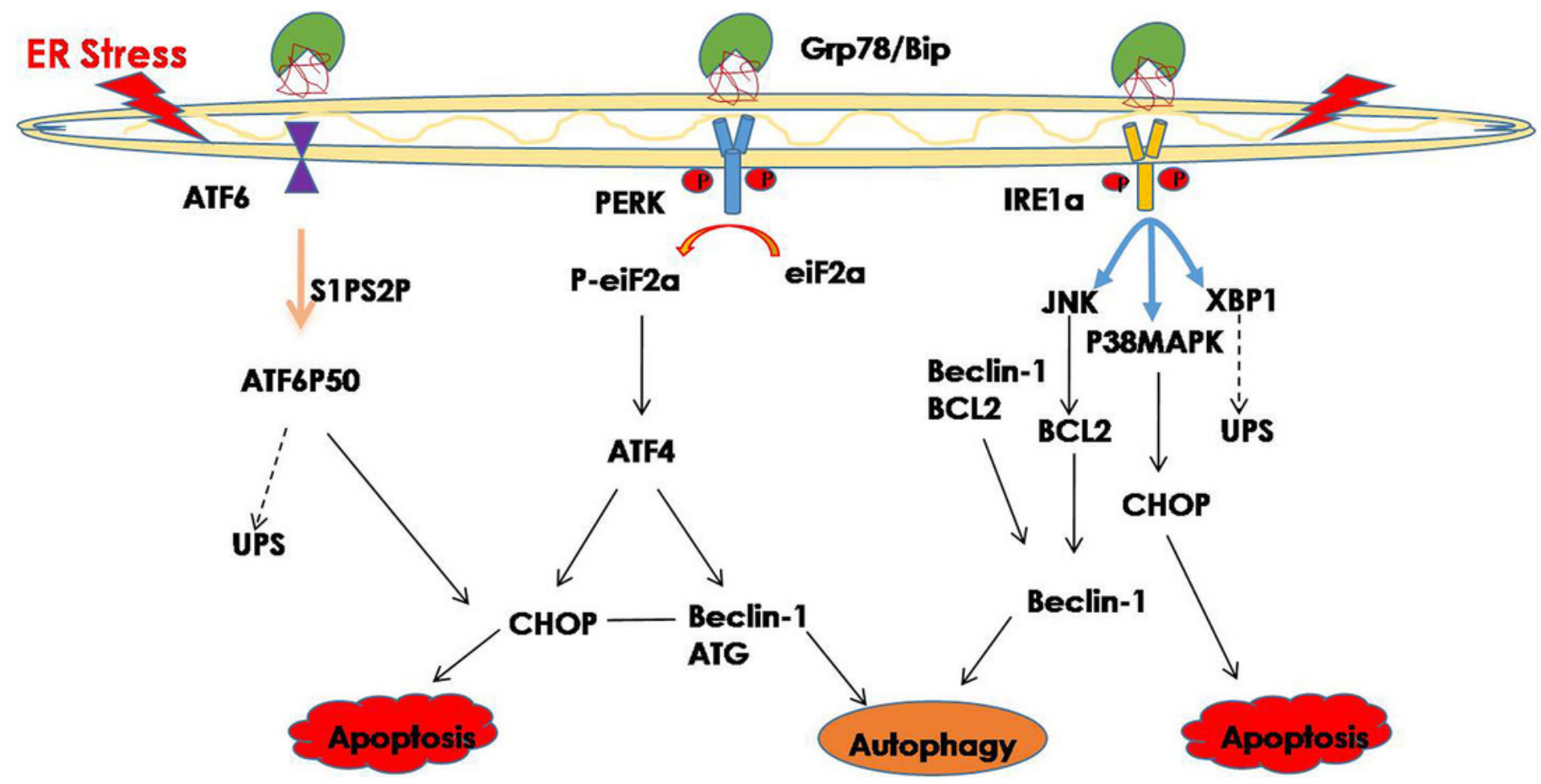




\section{Figure 2}

ER homeostasis can be achieved by balancing the UPS and autophagy pathways during ER stress.

During ER stress, the UPS and autophagy will be activated to remove harmful substrates such as misfolded proteins or protein aggregates to maintain the normal function of the ER. The balance between the UPS and autophagy is extremely important for restoring cell homeostasis.

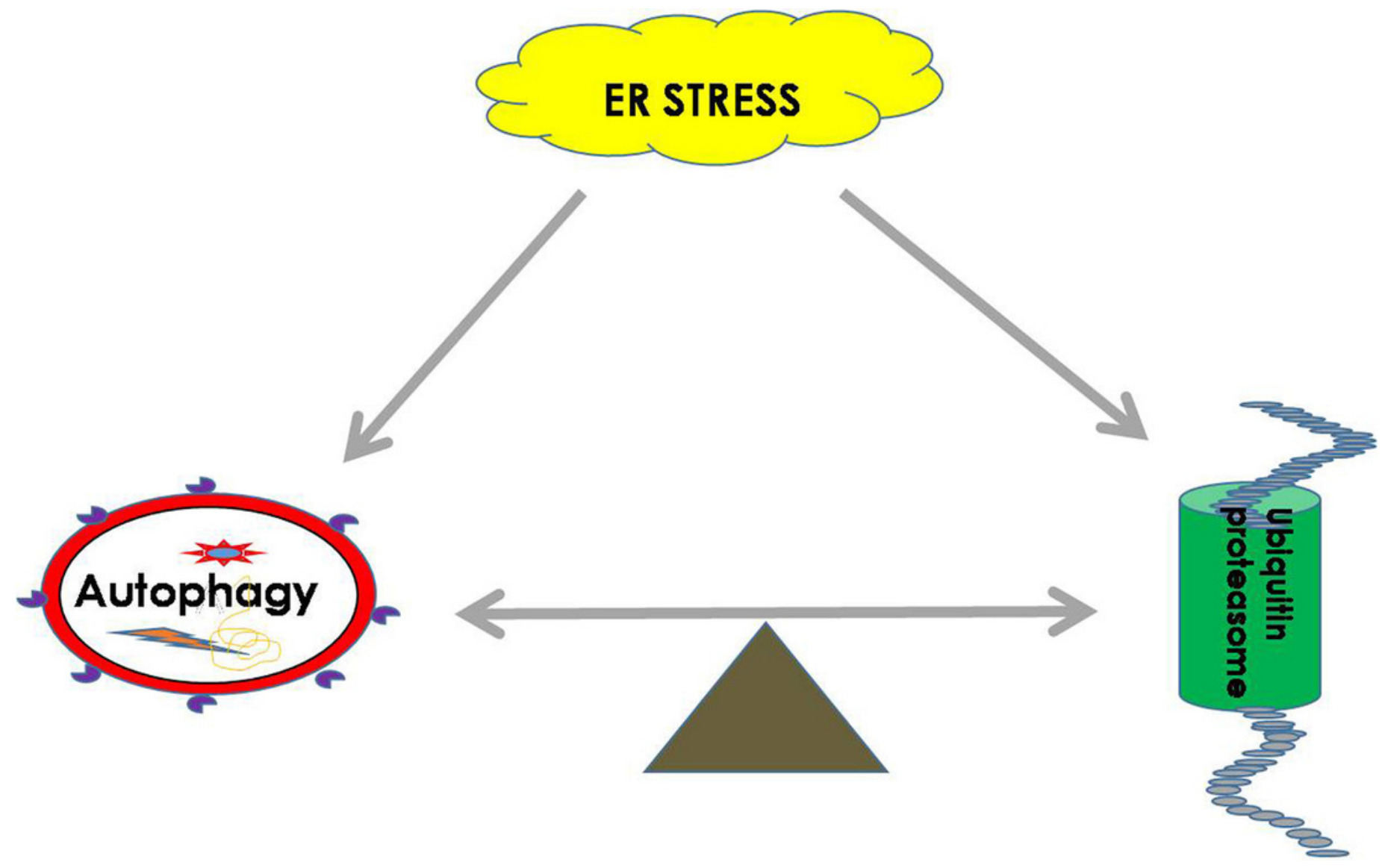

